\title{
Singlet Orbital Ordering in Bilayer $\mathrm{Sr}_{3} \mathrm{Cr}_{2} \mathrm{O}_{7}$
}

Justin Jeanneau, ${ }^{1,2}$ Pierre Toulemonde, ${ }^{1,2}$ Gyorgy Remenyi, ${ }^{1,2}$ André Sulpice, ${ }^{1,2}$ Claire Colin, ${ }^{1,2}$ Vivian Nassif, ${ }^{1,2,3}$ Emmanuelle Suard, ${ }^{3}$ Eduardo Salas Colera, ${ }^{4,5}$ Germán R. Castro, ${ }^{4,5}$ Frederic Gay, ${ }^{1,2}$ Corina Urdaniz, ${ }^{6,7}$ Ruben Weht, ${ }^{6,7,8}$ Clement Fevrier, ${ }^{1,2}$ Arnaud Ralko, ${ }^{1,2}$ Claudine Lacroix, ${ }^{1,2}$ Armando A. Aligia, ${ }^{7,9}$ and Manuel Núñez-Regueiro ${ }^{1,2, *}$

${ }^{1}$ Université Grenoble-Alpes, Institut Néel, 25 Avenue des Martyrs-BP166, 38042 Grenoble, cedex 9 France

${ }^{2}$ Centre Nationale de la Recherche Scientifique, Institut Néel, 25 Avenue des Martyrs-BP166, 38042 Grenoble, cedex 9 France

${ }^{3}$ Institut Laue -Langevin, 71 Avenue des Martyrs, 38000 Grenoble cedex 9 France

${ }^{4}$ European Synchrotron Radiation Facility, 71 rue des Martyrs, 38000 Grenoble, France

${ }^{5}$ Instituto de Ciencias de Materiales de Madrid-ICMM-CSIC, Sor Juana Inés de la Cruz, 3, 28049 Madrid, Spain

${ }^{6}$ Gerencia de Investigación y Aplicaciones, Comisión Nacional de Energía Atómica (CNEA),

Avda General Paz y Constituyentes, 1650 San Martín, Argentina

${ }^{7}$ Consejo Nacional de Investigaciones Científicas y Técnicas (CONICET), Buenos Aires, Argentina

${ }^{8}$ Instituto Sabato, Universidad Nacional de San Martín-CNEA, 1650 San Martín, Argentina

${ }^{9}$ Centro Atómico Bariloche and Instituto Balseiro, CNEA, 8400 Bariloche, Argentina

(Received 30 October 2016; published 19 May 2017)

We perform an extensive study of $\mathrm{Sr}_{3} \mathrm{Cr}_{2} \mathrm{O}_{7}$, the $n=2$ member of the Ruddlesden-Popper $\mathrm{Sr}_{n+1} \mathrm{Cr}_{n} \mathrm{O}_{3 n+1}$ system. An antiferromagnetic ordering is clearly visible in the magnetization and the specific heat, which yields a huge transition entropy, $R \ln (6)$. By neutron diffraction as a function of temperature we have determined the antiferromagnetic structure that coincides with the one obtained from density functional theory calculations. It is accompanied by anomalous asymmetric distortions of the $\mathrm{CrO}_{6}$ octahedra. Strong coupling and Lanczos calculations on a derived Kugel-Khomskii Hamiltonian yield a simultaneous orbital and moment ordering. Our results favor an exotic ordered phase of orbital singlets not originated by frustration.

DOI: 10.1103/PhysRevLett.118.207207

The interplay between spin and orbital degrees of freedom has been known for a long time [1,2]. Because of the large local Coulomb interactions, a low energy Hilbert space splits off, with superexchange couplings between both spin and orbital local degrees of freedom. The lattice may be distorted as a consequence of orbital ordering, although in many cases the situation is more complex [3]. The prototypical case is that of the perovskite $\mathrm{KCuF}_{3}$, where an antiferromagnetic spin order coexists with an antiferro-orbital order [1,4,5]. Potentially, the $\mathrm{Cr}$ based Ruddlesden-Popper (RP) series $\mathrm{Sr}_{n+1} \mathrm{Cr}_{n} \mathrm{O}_{3 n+1}$ are ideal candidates for investigating such phenomena. Unfortunately, they usually present so many synthesizing difficulties that research has been centered on the perovskite $\mathrm{SrCrO}_{3}$, the $n=\infty$ member of the family. As for many other transition metal oxides standard band structure calculations on $\mathrm{SrCrO}_{3}$ yield a metal [6], while published measurements on bulk polycrystalline $\mathrm{SrCrO}_{3}$ samples demonstrate a nonmetallic behavior $[7,8]$. Neutrondiffraction and synchrotron powder x-ray diffraction studies [9] showed that at $\mathrm{TN}=40 \mathrm{~K}$ a transition from a nonmagnetic cubic to an antiferromagnetic (AFM) tetragonal structure occurred. In the low temperature phase an orbital reoccupation $\left(d_{x y} d_{x z} d_{y z}\right)^{2} d_{x y}^{1}\left(d_{x z} d_{y z}\right)^{1}$ was found and explained by LDA $+\mathrm{U}$ calculations [6] showing that the distorted phase is coupled to a partial orbital ordering.

Because of their isolated biplane structure, the $n=2 \mathrm{RP}$ elements often show more complex physics, e.g. nematic orbital ordering in $\mathrm{Sr}_{3} \mathrm{Ru}_{2} \mathrm{O}_{7}$ [10] or antiferro-orbital (AFO) ordering accompanied by the formation of singlet spin dimers [11] in $\mathrm{K}_{3} \mathrm{Cu}_{2} \mathrm{~F}_{7}$. We thus studied $\mathrm{Sr}_{3} \mathrm{Cr}_{2} \mathrm{O}_{7}$ both on experimental and theoretical grounds in order to characterize its transitions. We have performed transport, magnetic, and specific heat measurements together with $\mathrm{x}$ rays and neutrons diffraction as a function of temperature. Our measurements determine simultaneous orbital and spin orderings at $210 \mathrm{~K}$, accompanied by anomalous asymmetric octahedra elongations. In order to understand the orbital ordering, we analyze the electronic properties through $a b$ initio calculations, which are complemented by the determination of superexchange interactions in strong coupling and Lanczos diagonalization of the resulting Kugel-Khomskii-type Hamiltonian. Our work shows that chromates are an interesting playground for orbital and spin degrees of freedom that will motivate more research of their promising properties.

Our polycrystalline samples were synthesized using a high-pressure-high-temperature method [7,12]. The Rietveld refinement performed on our x-ray patterns shows that our sample contains $94 \%$ of $\mathrm{Sr}_{3} \mathrm{Cr}_{2} \mathrm{O}_{7}, 4 \%$ of $\mathrm{SrCrO}_{3}$, and $2 \%$ of $\mathrm{Cr}_{2} \mathrm{O}_{3}$ (see the Supplemental Material [13]). Atomic positions and lattice parameters at ambient temperature $(a \simeq 3.82 \AA$ and $c \simeq 20.14 \AA)$ are in agreement with those obtained by Castillo et al. [7] (Table I of Supplemental Material [13]). Electrical resistivity and 


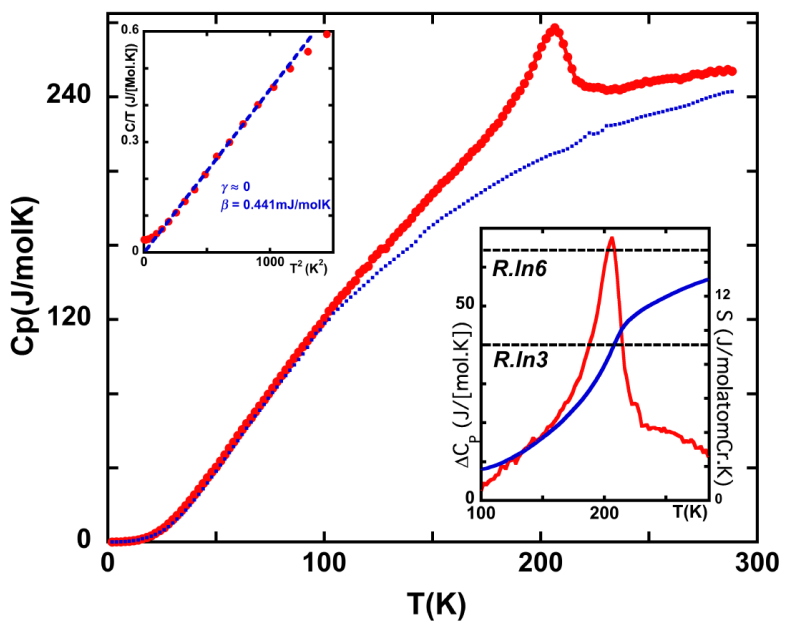

FIG. 1. Specific heat of $\mathrm{Sr}_{3} \mathrm{Cr}_{2} \mathrm{O}_{7}$ (red line) and measured specific heat of $\mathrm{Sr}_{3} \mathrm{Ti}_{2} \mathrm{O}_{7}$ baseline (blue squares) (see Supplemental Material [13]). Left inset: low temperature specific heat showing that $\gamma=0$, i.e. no free carriers at $T=0$, confirming the nonmetallic electrical resistivity behavior (see Supplemental Material [13]). Right inset: excess specific heat (red line) and entropy of transition (blue line) as a function of temperature.

magnetization measurements (see the Supplemental Material [13]) detect the AFM transition [7] with $T_{N}=210 \mathrm{~K}$.

Thermal properties are key factors in determining the degrees of freedom frozen at each transition. We thus performed the specific heat measurements on $\mathrm{Sr}_{3} \mathrm{Cr}_{2} \mathrm{O}_{7}$ that are shown in Fig. 1. We observe a huge anomaly at $T_{N}$, implying a large transition entropy. To evaluate it, we take as the phonon contribution the specific heat of the similar but nonmagnetic compound $\mathrm{Sr}_{3} \mathrm{Ti}_{2} \mathrm{O}_{7}$, which we synthesized and measured. From the excess specific heat we calculate the transition entropy per Cr mole. $\mathrm{In}_{\mathrm{Sr}_{3}} \mathrm{Cr}_{2} \mathrm{O}_{7}$, the formal oxidation state of the $\mathrm{Cr}$ ions is $4+$ with two electrons accommodated in the $t_{2 g}$ manifold. Hund's rules favor parallel spins within each manifold, and lead to spin $S=1$. If the transition is accompanied by magnetic ordering only, $\mathrm{Cr}$ ions are expected to contribute $R \ln (3)$ to the entropy change. We observe a value near $R \ln (5)$, that, considering the difficult synthesizing conditions of this sample and the impurity phases, would imply actually a $R \ln (6)$ value. Our entropy value is anomalously high, since in many magnetic or orbital transitions only around $20 \%$ of the expected entropy change is detected (e.g. $\mathrm{KCuF}_{3}$ [27]). Thus, this result calls undoubtedly for a full magnetic and orbital ordering at $T_{N}$.

The next step to characterize the magnetic and orbital transitions is to determine the AFM order and the precise oxygen positions above and below the complex phase transition at $210 \mathrm{~K}$. We thus performed neutron powder diffraction at five different temperatures: $2 \mathrm{~K}, 50 \mathrm{~K}, 150 \mathrm{~K}$, $300 \mathrm{~K}$ at D1B/ILL to detect the magnetic peaks and at $\mathrm{D} 2 \mathrm{~B} / \mathrm{ILL}$ to refine the crystallographic structure.
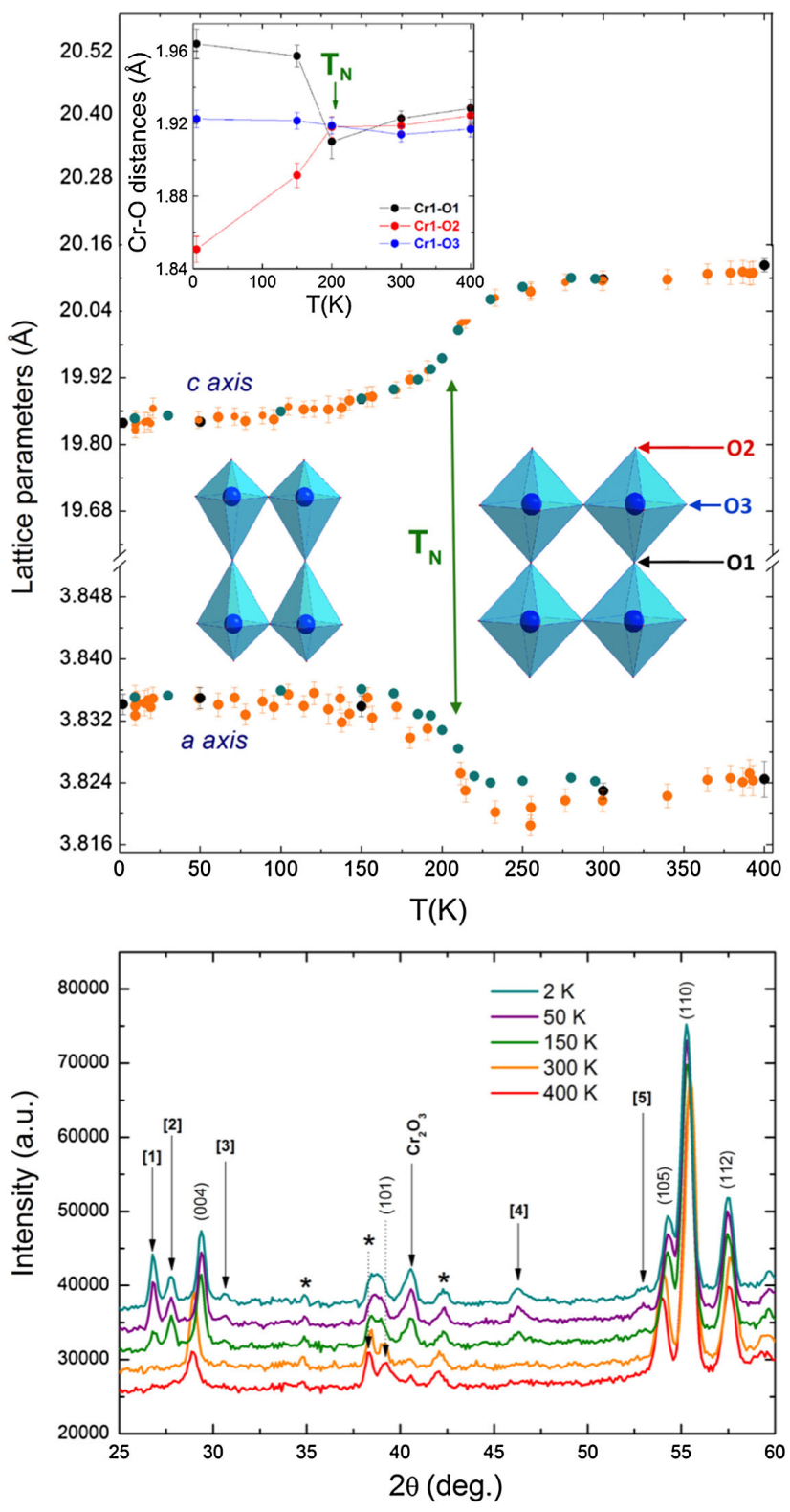

FIG. 2. Upper panel: variation of the lattice parameters as a function of temperature of $\mathrm{Sr}_{3} \mathrm{Cr}_{2} \mathrm{O}_{7}$ (orange circles: XRD; green squares: neutrons). Upper inset: variation of the $\mathrm{Cr}$-apical $\mathrm{O}$ distances as a function of temperature. Middle inset: schematic deformation of the structure, with a clear separation of the twin $\mathrm{CrO}_{2}$ layers. Lower panel: neutron diffraction spectra for $\mathrm{Sr}_{3} \mathrm{Cr}_{2} \mathrm{O}_{7}$ at different temperatures, showing the appearing of the numbered magnetic peaks at $300 \mathrm{~K}$. The calculated magnetic structure at $2 \mathrm{~K}$ is shown in the Supplemental Material [13] and coincides with the second one in Fig. 3.

We present the D1B/ILL measurements on the lower panel of Fig. 2. We can note on the neutron diffraction profile five magnetic contributions all appearing between 300 and $150 \mathrm{~K}$. These temperature values are in agreement with $T_{N}=210 \mathrm{~K}$. Rietveld fitting of our data at $2 \mathrm{~K}$ (see the Supplemental Material [13] for more details) yields a magnetic solution with slanted moments of $1.34 \mu_{B}$ AFM 
ordered within the planes and FM between the planes (Fig. 3, second structure).

The variation of cell parameters as a function of temperature, extracted both from x-ray diffraction (XRD) and neutrons is shown on the upper panel of Fig. 2. We observe that, as a function of temperature, there is a contraction of the $c$ parameter and a dilatation of the $a$ parameter at $T_{N}$. The low temperature structure shows an unexpected deformation. While the octahedra are almost regular above $T_{N}$, at low temperatures there is a separation of the $\mathrm{CrO}_{2}$ planes of the bilayer with a contraction of the distance between the outer apical oxygen $(\mathrm{O} 2)$ and the chromium, schematically shown in the middle panel of Fig. 2.

We now describe our theoretical results on $\mathrm{Sr}_{3} \mathrm{Cr}_{2} \mathrm{O}_{7}$, in order to understand the origin of its magnetic ordering and the nature of the orbital ordering and its deformations. From our nonmagnetic density functional theory (DFT) calculation we obtained that $\mathrm{Sr}_{3} \mathrm{Cr}_{2} \mathrm{O}_{7}$ is, at this level, metallic, with narrow $t_{2 g}$ states around the Fermi level $\left(\mathrm{E}_{F}\right)$. These states have $d_{x y}$ and $d_{x z} / d_{y z}$ characters, with $d_{z^{2}}$ and $d_{x^{2}-y^{2}}$ orbitals slightly higher in energy (Fig. 3, upper panel). Doing the projection on Wannier functions (see Methods in the Supplemental Material [13]) we obtained that the $d_{x y}$ level is around $50 \mathrm{meV}$ lower in energy than the $d_{x z} / d_{y z}$ states. Strong peaks in the density of states around $\mathrm{E}_{F}$ makes the material close to a magnetic instability. To check for the relative stability of the different magnetic
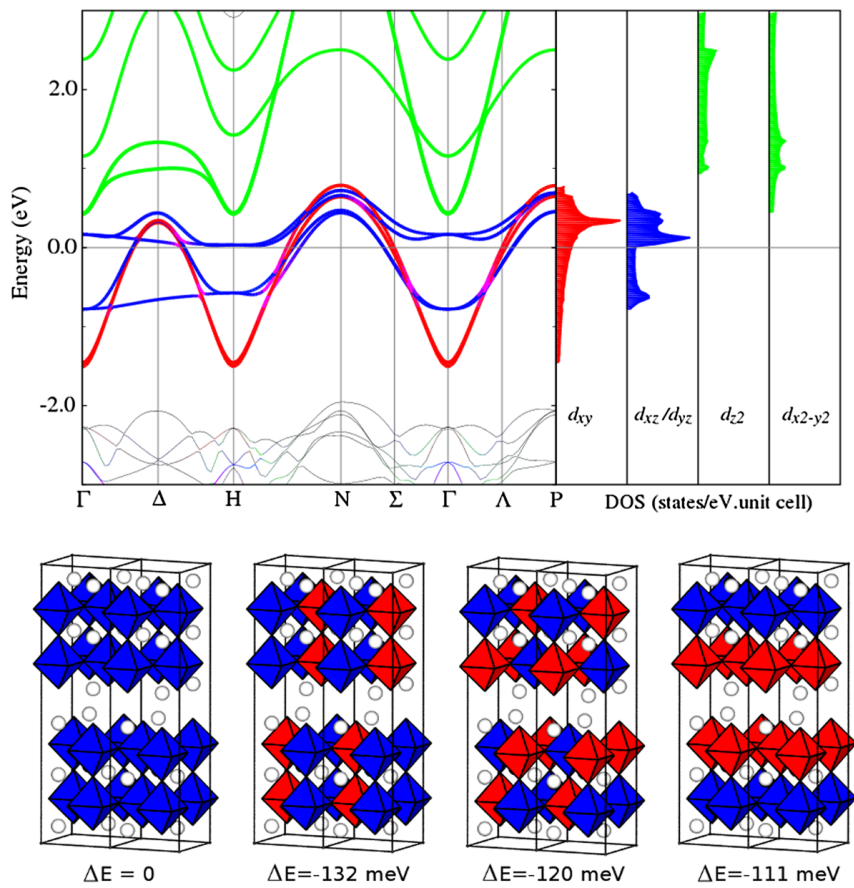

FIG. 3. Upper panel: band structure and DOS for the nonmagnetic structure. Lower panel: energies for the different magnetic configurations we have considered. Different colors, different moment orientations. Values are referred to the completely ferromagnetic solution. solutions, we have carried out a series of calculations considering a ferromagnetic and three different antiferromagnetic configurations within each bilayer, represented in Fig. 3, lower panel. The most stable configuration is antiferromagnetic within each layer but ferromagnetically coupled between layers, in agreement with the results obtained by neutron experiments mentioned before. That system is still metallic but with much smaller bandwidth. All the magnetic configurations result in local magnetic moments of $\simeq 1.5$ Bohr magneton in accordance with the expected $S=1$ configuration. Conduction bands are quite flat, suggesting that any impurity or imperfection can easily influence conduction measurements. As the bandwidth is quite small and the correlations can be very important for this kind of oxides, we made a DFT $+U$ series of calculations with different effective Hubbard U parameters. The calculations did not change the relative stability of the magnetic phases, but made the bands still flatter, particularly for the conduction states. As in Ref. [6], we obtained an insulating solution with a critical $\mathrm{U}$ of around $3 \mathrm{eV}$.

As in $\mathrm{SrCrO}_{3}$ [6] we obtain the ground-state configuration $d_{x y}^{1}\left(d_{x z} d_{y z}\right)^{1}$. Therefore, because of Hund's rules, one expects that the ground state of isolated $\mathrm{Cr}^{+4}$ atoms is sixfold degenerate and consists of two $S=1$ spin triplets, with one hole occupying the $d_{x y}$ orbital and the other one occupying either the $d_{y z}$, or the $d_{x z}$ orbital. In fact the entropy $R \ln (6)$ associated with the transition observed at $210 \mathrm{~K}$ points to a simultaneous ordering of the spin and orbital degrees of freedom.

The eigenstates of the 3D shell for a few electrons including all interactions have been calculated, for example, in Ref. [28]. To go beyond isolated $\mathrm{Cr}$ atoms, we have calculated the effect of Cr-O hopping in fourth order perturbation theory. We have derived an effective model of the Kugel-Khomskii type for two neighboring $\mathrm{CrO}$ planes with effective spin and orbital interactions, neglecting interactions between $\mathrm{Cr}$ atoms in different bilayers (see details in the Supplementary Material [13]). In this compound, the local spin is $S_{i}=1$, while the pseudospin is $T_{i}=1 / 2$ since the orbital degeneracy is twofold.

The effective Hamiltonian can be written as

$$
H_{\mathrm{eff}}=\sum_{k} H_{k}+\sum_{j} H_{j}^{12}
$$

where $H_{k}, k=1,2$, contains the interactions within the plane $k$, and $H_{j}^{12}$ describes the interactions between $\mathrm{Cr}$ sites in different planes at the same two-dimensional position $j$. Two neighboring $\mathrm{Cr}$ atoms, in any $\alpha$ direction $(\alpha=x, y$ or $z$ ) have an $\mathrm{O}$ in between. The superexchange interaction between electrons in $t_{2 g}$ orbitals of neighboring $\mathrm{Cr}$ sites is mediated by $p$ orbitals of this $\mathrm{O}$ atom, as indicated in Figs. 4(a) and 4(b). In the $z$ direction, only the $x z$ and $y z$ orbitals contribute to this interaction and similarly in the $x$ and $y$ directions. Collecting the different terms, and 
(a)

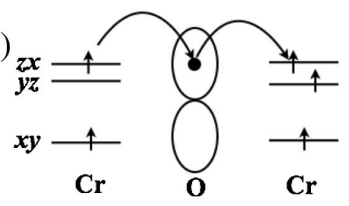

(b)

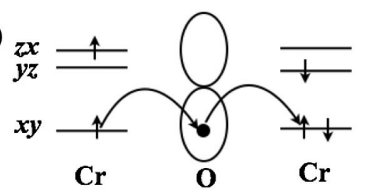

(c)

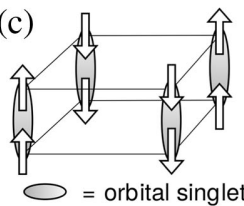

(d)

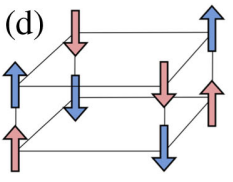

(e)
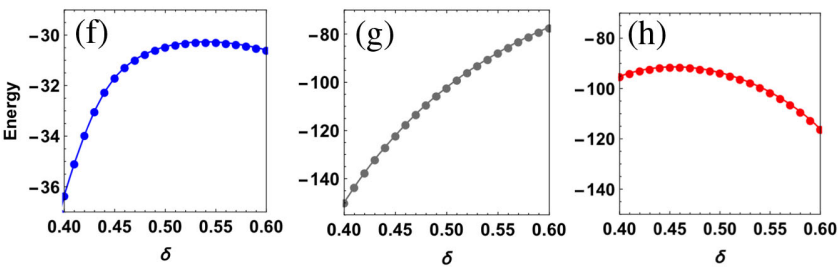

FIG. 4. (a) Scheme of the dominant fourth-order perturbation term in the $z$ direction. The arrows indicate the spin of the occupied $\mathrm{Cr}$ orbitals after the first hop from an $\mathrm{O}$ atom (middle) to a $\mathrm{Cr}$ atom (right). (b) Scheme of the contribution of $x y$ orbitals to the Heisenberg interaction in the $x y$ plane. (c)-(e): Three different phases with a spin structure compatible (arrows) observed experimentally: antiferromagnetic in the planes and ferromagnetic between the layers. The color of the arrow distinguishes the orbital. The orbital pseudospins (c) are paired as singlets (gray ovals) along the outplane bonds, (d) have the same antiferro-orbital correlations in and out of the planes, and (e) are ferro-orbital in the planes and antiferro-orbital out of the planes. (f)-(h): evolution of the energy for each of these phases as a function of the parameter $\delta$ that describes the crystal field.

dropping an irrelevant constant, we obtain for the interplane interaction:

$H_{j}^{12}=I_{S} \mathbf{S}_{1 j} \cdot \mathbf{S}_{2 j}+I_{T} \mathbf{T}_{1 j} \cdot \mathbf{T}_{2 j}+I_{S T}\left(\mathbf{T}_{1 j} \cdot \mathbf{T}_{2 j}\right)\left(\mathbf{S}_{1 j} \cdot \mathbf{S}_{2 j}\right)$,

where $\mathbf{S}_{k j}\left(\mathbf{T}_{k j}\right)$ is the spin (pseudospin) at a site of the plane $k$ with position $j$ (see the Supplemental Material [13] for details and expressions of the interactions $I_{T}, I_{S T}$, and $I_{S}$ in terms of the microscopic parameters of the compound). Similarly it is possible to get the effective in-plane superexchange Hamiltonian $H_{k}$, whose expression is more complicated and given in the Supplemental Material [13].

We have approached the problem numerically by diagonalizing the microscopic Hamiltonian Eq. (1) using a Lanczos algorithm on a $2 \times 2$ bilayer in the complete parameter space (see the Supplemental Material [13]). By doing a systematic analysis of the spin-spin $\left\langle\mathbf{S}_{i} \cdot \mathbf{S}_{j}\right\rangle$ and orbital-orbital $\left\langle\mathbf{T}_{i} \cdot \mathbf{T}_{j}\right\rangle$ real space correlation functions, we have found a very rich phase diagram with more than seven phases (see the Supplemental Material [13] for details). Among these phases, only three have a spin structure compatible with the magnetic order observed experimentally, namely antiferromagnetic in the planes and ferromagnetic out of the planes. These three phases are displayed in Figs. 4(c)-(e). Among these phases, we have found an original orbital dimer phase in which all orbitals of the vertical bounds are paired as singlets.

As shown in Fig. 2, the $\mathrm{CrO}_{2}$ planes separate as the temperature is lowered. In order to understand the reason of this unexpected distortion, we have looked at the effect of the crystalline field that enters in our model via the parameter $\delta$ (the splitting between the degenerate $x z$ and $y z$ orbitals and the $x y$ one; see the Supplemental Material [13]) on the energy stability by scanning around a realistic value set at $\delta=0.5$, as shown in Fig. 4. If the energy lowers as $\delta$ decreases, then the system will tend to separate the two layers, as the crystalline field is expected to decrease with the distance between the planes. This is precisely what is happening for two of our compatible solutions, (c) and (d). For the most exotic dimer phase (c), it is quite counterintuitive that a singlet bound state would not drive to contraction instead. In fact, looking closely to the energy around $\delta=0.5$, we see that the system is very close to a saddle point at $\delta_{c}=0.55$ at which the system will enter a normal contracting deformation as observed, e.g., in spin systems if $\delta<\delta_{c}$, or the present dilatation if $\delta>\delta_{c}$. Thus, both the orbital singlet, Fig. 4(c), and the totally antiferroorbital order, Fig. 4(d), may explain the observed plane separation at low $T$.

Experimentally, the latter should give an in-plane distortion, as in $\mathrm{K}_{2} \mathrm{CuF}_{4}$ for example [29]. We have tried to observe it with high resolution XRD and EXAFS experiments at ESRF. However, within our precision, there is no such distortion, favoring the orbital singlet phase (see the Supplemental Material [13]). $\mathrm{Sr}_{3} \mathrm{Cr}_{2} \mathrm{O}_{7}$ would thus be similar to $\mathrm{K}_{3} \mathrm{Cu}_{2} \mathrm{~F}_{7}$, with an exchange of roles between the orbital and spin degrees of freedom. In $\mathrm{K}_{3} \mathrm{Cu}_{2} \mathrm{~F}_{7}$ the existence of magnetic spin singlets has been attributed to spin-orbital entanglement [30]. Here we can propose that similar entanglement for $\mathrm{Sr}_{3} \mathrm{Cr}_{2} \mathrm{O}_{7}$ leads to the formation of interlayer orbital singlets.

In conclusion, our extensive experimental and theoretical studies yield features that make the magnetic and orbital ordering of $\mathrm{Sr}_{3} \mathrm{Cr}_{2} \mathrm{O}_{7}$ anomalous. So far, orbital singlets have been only observed in frustrated systems where a orbital liquid can be realized [31]. Besides, in the present case, the orbital singlet formation is accompanied by a separation of the ions whose orbitals pair, indicating that pairing in this compound is analogous to a spin-Peierls dimerization, but in the orbital sector. The compound $\mathrm{YVO}_{3}$ [32] was also proposed to exhibit an orbital Peierls ground state or similarly an "orbital valence bond" state [33], but this was contradicted by further studies [34-36] that proposed a usual orbital order for this system. For $\mathrm{Sr}_{3} \mathrm{Cr}_{2} \mathrm{O}_{7}$, further experimental studies have to be performed to confirm our proposal. In particular, the coupling to the lattice should be different for conventional orbitalorder. 
J. J. is supported by the LANEF framework with mutualized infrastructure (ANR-10-LABX-51-01). We acknowledge support from CONICET (Grant No. PIP 114-20110100376 and 112-201101-00832) and ANPCyT (Grant No. PICT-2012-0609 and PICT-2013-1045).

*Corresponding author. nunez@neel.cnrs.fr

[1] K. I. Kugel and D. I. Khomskii, Sov. Phys. Usp. 25, 231 (1982).

[2] M. Cyrot and C. Lyon-Caen, J. Phys. (Les Ulis, Fr.) 36, 253 (1975).

[3] L. F. Feiner, A. M. Olés, and J. Zaanen, Phys. Rev. Lett. 78, 2799 (1997).

[4] L. G. Marshall, J. Zhou, J. Zhang, J. Han, Sven C. Vogel, Y. Zhao, M. T. Fernandez-Diaz, X. Yu, J. Cheng, and J. B. Goodenough, Phys. Rev. B 87, 014109 (2013).

[5] A. Okazaki and Y. Suemune, J. Phys. Soc. Jpn. 16, 176 (1961).

[6] K.-W. Lee and W. E. Pickett, Phys. Rev. B 80, 125133 (2009).

[7] E. Castillo-Martinez and M. A. Alario Franco, Solid State Sci. 9, 564 (2007).

[8] J.-S. Zhou, C.-Q. Jin, Y.-W. Long, L.-X. Yang, and J. B. Goodenough, Phys. Rev. Lett. 96, 046408 (2006).

[9] L. Ortega-San-Martin, A. J. Williams, J. Rodgers, J. P. Attfield, G. Heymann, and H. Huppertz, Phys. Rev. Lett. 99, 255701 (2007).

[10] C. Stingl, R. S. Perry, Y. Maeno, and P. Gegenwart, Phys. Rev. Lett. 107, 026404 (2011).

[11] H. Manaka, Y. Miyashita, Y. Watanabe, and T. Masuda, J. Phys. Soc. Jpn. 76, 044710 (2007).

[12] J. A. Kafalas and J. M. Longo, J. Solid State Chem. 4, 55 (1972).

[13] See Supplemental Material at http://link.aps.org/ supplemental/10.1103/PhysRevLett.118.207207, which includes Refs. [14-26], for details about the synthesis and structural characterization of the system, LDA calculations and derivation of the effective Hamiltonian and its solution.

[14] S. N. Ruddlesden and P. Popper, Acta Crystallogr. 11, 54 (1958).

[15] J. Rodriguez-Carvajal, Physica B (Amsterdam) 192, 55 (1993); J. Rodriguez-Carvajal, in Commission on Powder Diffraction (IUCr), (Newsletter 2001), Vol. 26, pp. 12-19, https://www.fkf.mpg.de/4112052/cpd26.pdf.

[16] P. Hohenberg and W. Kohn, Phys. Rev. 136, B864 (1964); W. Kohn and L. J. Sham, Phys. Rev. 140, A1133 (1965).
[17] P. Blaha, K. Schwarz, G. K. H. Madsen, D. Kvasnicka, and J. Luitz, WIEN2K (Technische Universitt Wien, Austria, 2002).

[18] G. Kresse and J. Hafner, Phys. Rev. B 47, 558 (1993); 49, 14251 (1994); G. Kresse and J. Furthmüller, Comput. Mater. Sci. 6, 15 (1996); Phys. Rev. B 54, 11169 (1996).

[19] J. P. Perdew, A. Ruzsinszky, G. I. Csonka, O. A. Vydrov, G. E. Scuseria, L. A. Constantin, X. Zhou, and K. Burke, Phys. Rev. Lett. 100, 136406 (2008); 102, 039902(E) (2009).

[20] J. P. Perdew, K. Burke, and M. Ernzerhof, Phys. Rev. Lett. 77, 3865 (1996).

[21] N. Marzari and D. Vanderbilt, Phys. Rev. B 56, 12847 (1997); N. Marzari, A. A. Mostofi, J. R. Yates, I. Souza, and D. Vanderbilt, Rev. Mod. Phys. 84, 1419 (2012).

[22] A. C. Komarek et al., Phys. Rev. B 84, 125114 (2011).

[23] A. J. Williams, A. Gillies, J. P. Attfield, G. Heymann, H. Huppertz, M. J. Martínez-Lope, and J. A. Alonso, Phys. Rev. B 73, 104409 (2006).

[24] A. M. Arevalo-Lopez, B. Liang, M. S. Senn, C. Murray, C. Tang, and J.P. Attfield, J. Mater. Chem. C 2, 9364 (2014).

[25] P. G. DeGennes and J. Friedel, J. Phys. Chem. Solids 4, 71 (1958).

[26] M. Imada, A. Fujimori, and Y. Tokura, Rev. Mod. Phys. 70, 1039 (1998).

[27] K. Hirakawa, J. Yoshinaga, and H. Miike, J. Phys. Soc. Jpn. 36, 906 (1974).

[28] A. A. Aligia and M. A. Gusmão, Phys. Rev. B 70, 054403 (2004).

[29] T. Ito and J. Akimitsu, J. Phys. Soc. Jpn. 40, 1333 (1976).

[30] W. Brzezicki and A. M. Oles, J. Phys. Conf. Ser. 391, 012085 (2012).

[31] B. Normand and A. M. Olés, Phys. Rev. B 78, 094427 (2008).

[32] C. Ulrich, G. Khaliullin, J. Sirker, M. Reehuis, M. Ohl, S. Miyasaka, Y. Tokura, and B. Keimer, Phys. Rev. Lett. 91, 257202 (2003).

[33] P. Horsch, G. Khaliullin, and A. M. Olés, Phys. Rev. Lett. 91, 257203 (2003).

[34] Z. Fang and N. Nagaosa, Phys. Rev. Lett. 93, 176404 (2004).

[35] M. De Raychaudhury, E. Pavarini, and O. K. Andersen, Phys. Rev. Lett. 99, 126402 (2007).

[36] S. Sugai and K. Hirota, Phys. Rev. B 73, 020409(R) (2006). 